\title{
State and fault estimation based on fuzzy observer for a class of Takagi-Sugeno singular models
}

\author{
Kaoutar Ouarid ${ }^{1,2}$, Mohamed Essabre ${ }^{3}$, Abdellatif El Assoudi ${ }^{1,2}$, El Hassane El Yaagoubi ${ }^{1,2}$ \\ ${ }^{1}$ Laboratory of High Energy Physics and Condensed Matter, Faculty of Science Ain Chock, Hassan II University of Casablanca, \\ Casablanca, Morocco \\ ${ }^{2}$ ECPI, Department of Electrical Engineering, ENSEM, Hassan II University of Casablanca, Casablanca, Morocco \\ ${ }^{3}$ Laboratory of Condensed Matter Physics and Renewable Energy, Faculty of Sciences and Technologies Mohammedia, \\ Hassan II University of Casablanca, Casablanca, Morocco
}

\begin{abstract}
Article Info
Article history:

Received Jun 23, 2021

Revised Oct 23, 2021

Accepted Nov 30, 2021

\section{Keywords:}

Fault diagnosis

Fuzzy observer

LMIs

Lyapunov theory

Takagi-Sugeno singular model

ABSTRACT

Singular nonlinear systems have received wide attention in recent years, and can be found in various applications of engineering practice. On the basis of the Takagi-Sugeno (T-S) formalism, which represents a powerful tool allowing the study and the treatment of nonlinear systems, many control and diagnostic problems have been treated in the literature. In this work, we aim to present a new approach making it possible to estimate simultaneously both non-measurable states and unknown faults in the actuators and sensors for a class of continuous-time Takagi-Sugeno singular model (CTSSM). Firstly, the considered class of CTSSM is represented in the case of premise variables which are non-measurable, and is subjected to actuator and sensor faults. Secondly, the suggested observer is synthesized based on the decomposition approach. Next, the observer's gain matrices are determined using the Lyapunov theory and the constraints are defined as linear matrix inequalities (LMIs). Finally, a numerical simulation on an application example is given to demonstrate the usefulness and the good performance of the proposed dynamic system.
\end{abstract}

This is an open access article under the CC BY-SA license.

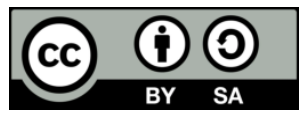

\section{Corresponding Author:}

Kaoutar Ouarid

Laboratory of High Energy Physics and Condensed Matter, Faculty of Science Ain Chock

Hassan II University of Casablanca

Km 8 Road El Jadida, B.P 5366 Maarif, 20100, Casablanca 20000, Morocco

Email: kaoutar.ouarid@gmail.com

\section{INTRODUCTION}

Over the last decades, the increase in the performance of equipment in terms of production quality and gain in productivity was accompanied by the complexity of the equipment. However, the presence of abnormal changes due to actuator or system or sensor faults can degrade system performances, hence the need to integrate fault detection and diagnosis (FDD) tools [1], [2] to maintain, for a long time, the desired performance of the whole system in various sectors. In particular, FDD has a very important role in monitoring the behavior of system variables and revealing faults, and it is performed based on the relative information to the system and its equipment. This information can be obtained by adding sensors to acquire measured states or observers to estimate non-measured states requiring expensive or difficult sensors to maintain. The state modeling of process dynamics is often obtained based on its state variables linked together by mathematical equations. If these processes have constraints, then it is necessary to use static equations to sufficiently characterize the studied process. Such systems composed of static and dynamic equations are called singular, or descriptor or implicit systems [3]. Recently, the FDD problem for singular 
systems has attracted much attention in various fields such as mechanical engineering, computer science, civil engineering, electrical engineering and automation.

Various techniques for detecting and estimating faults have been proposed for the class of linear systems [4], [5], and for the class of nonlinear systems [6]-[8] allowing to provide a closer representation to the real system, but which are difficult to exploit. Due to this complexity, it has become essential to work with a precise class of nonlinear systems such as Lipschitz systems, uncertain systems, bilinear systems or others. From these classes of nonlinear systems, we find the class of Takagi-Sugeno (T-S) [9] nonlinear systems, in ordinary or singular form. It has been introduced to compromise between the good precision of the nonlinear behavior of the studied system, and the use of techniques adapted to linear systems due to the convex sum property of its activation functions [3], [10].

There have been many methods of FDD [2], [11], [12] which can be classified into signal-based approaches [13], knowledge-based approaches [12], [14], and process model-based approaches [12], [13] which contain state observer-based method representing an analytical method having achieved several results in this field, and which depends on the mathematical model of the studied system without needing other components. Many publications have been interested in the design of observers for FDD [15]-[24] and have presented fruitful results. A residual generator for detecting and isolating actuator faults for a class of T-S fuzzy bilinear system is developed in [18]. Developing a novel fuzzy FD observer for FD of sensors faults of T-S fuzzy systems is the aim of the work presented in [19]. In [20], depicted a T-S unknown input observer to simultaneously estimate the interval of states and actuator faults for a class of T-S explicit systems. Another technique based on a robust fault estimation observer has been introduced for estimating actuator faults for a class of discrete-time singular systems [21]. In [22], a design of an adaptive observer is proposed for detecting sensor faults of an industrial servo system. For the fault diagnosis and reconstruction of the faults affecting the states of the system, in [23] suggested a new augmented linear parameter-varying (LPV) observer for a class of LPV models. The design of a combination of reduced-order LPV and full-order LPV unknown input observers, respectively, for FDD of actuator and sensor faults of industrial processes is presented in [24].

Most of these observers are synthesized to estimate only actuator or sensor faults while guaranteeing asymptotic convergence for various class of nonlinear system in continuous or discrete-time. The goal of our work is not to compare our approach with those already carried out, but rather to extend our results from the case of singular linear models [25] and T-S singular models with measurable premise variables [26] to the case of T-S singular models with unmeasurable premise variables while ensuring an exponential convergence, and simultaneously estimating the unmeasurable states and the faults at the level of actuators and sensors. In this work, for simultaneous estimation of states and faults, the novel suggested technique consists to associate for each local model a local observer. Then, the proposed fuzzy observer is obtained by an aggregation of the local observers. Our contribution is based on the separation of the dynamic equations from the static equations which makes it possible to facilitate and minimize the computation by obtaining the static states just from the dynamic states already found. The design conditions are expressed in terms of LMIs. This observer is applied for both actuators and sensors faults for a class of T-S singular model in the case of unmeasurable premise variables. The paper is composed of five parts that are presented as follows: Section 2 exposes the class of the studied system. Section 3 provides the synthesis of the proposed observer and the stability conditions. The numerical results of the application example are given in section 4. Section 5 is devoted to a brief conclusion.

\section{MATHEMATICAL FORMULATION OF THE CONSIDERED MODEL}

In this paper, the following class of continuous-time Takagi-Sugeno singular model (CTSSM) with unmeasurable premise variables in presence of actuator and sensor fault is considered (1),

$$
\left\{\begin{array}{c}
M \dot{z}=\sum_{i=1}^{q} \rho_{i}(\beta)\left(A_{i} z+B_{i} \tau+M_{a i} v_{a}\right) \\
y=\sum_{i=1}^{q} \rho_{i}(\beta)\left(C_{i} z+D_{i} \tau+D_{a i} v_{a}+M_{s i} v_{s}\right)
\end{array}\right.
$$

where $z=\left[Z_{1}^{T} Z_{2}^{T}\right]^{T} \in \mathbb{R}^{n}$ is the state vector with $Z_{1} \in \mathbb{R}^{r}$ is the vector of dynamic variables, $Z_{2} \in \mathbb{R}^{n-r}$ is the vector of static variables, $\tau \in \mathbb{R}^{p}$ is the control input, $y \in \mathbb{R}^{m}$ is the measured output vector, $v_{a} \in \mathbb{R}^{a}$ and $v_{s} \in \mathbb{R}^{b}$ are the actuator and sensor fault vectors, respectively. The matrices $A_{i}, B_{i}, M_{a i}, C_{i}, D_{i}, D_{a i}$ and $M_{s i}$ are real known constant matrices with adequate dimensions related with the $i^{\text {th }}$ local model with,

$$
A_{i}=\left(\begin{array}{ll}
A_{11 i} & A_{12 i} \\
A_{21 i} & A_{22 i}
\end{array}\right) ; B_{i}=\left(\begin{array}{l}
B_{1 i} \\
B_{2 i}
\end{array}\right) ; M_{a i}=\left(\begin{array}{c}
M_{a i}^{1} \\
M_{a i}^{2}
\end{array}\right) ; \quad C_{i}=\left(C_{1 i} C_{2 i}\right)
$$


the rank of the matrices $A_{22 i}$ are equal to $n-r$ and it is supposed to be invertible. $q$ represents the number of sub-models, and the premise variable $\beta$ is supposed to be real-time accessible.

$$
\left\{\begin{array}{c}
M \dot{z}=A_{i} z+B_{i} \tau+M_{a i} v_{a} \\
y=C_{i} z+D_{i} \tau+D_{a i} v_{a}+M_{s i} v_{s}
\end{array}\right.
$$

The transition between the contributions of each sub model (3) is ensured by the terms $\rho_{i}(\beta)$ which represent the weighting functions, depending on the states of the system and verifying the property of the convex sum,

$$
\sum_{i=1}^{q} \rho_{i}(\beta)=1 ; 0 \leq \rho_{i}(\beta) \leq 1 ; i=1, \ldots, q
$$

the matrix $M$ whose $\operatorname{rank}(M)=r<n$ is assumed to have the following form,

$$
M=\left(\begin{array}{ll}
I & 0 \\
0 & 0
\end{array}\right)
$$

Assumption 1: Assume that [3]:

$-\left(M, A_{i}\right)$ are regular, i.e. $\operatorname{det}\left(s M-A_{i}\right) \neq 0 \forall s \in \mathbb{C}$

- The sub-models (3) are impulse observable and detectable

The separation of the dynamic equations from the static equation in each sub-model ( 3 ) is the aim of our approach, and then the aggregation of the resulting sub-models allows obtaining the global fuzzy model. So, using the expression of the matrices (2) and (5), the sub-model (3) can be written in the following second equivalent form [3],

$$
\left\{\begin{array}{c}
\dot{Z}_{1}=A_{11 i} Z_{1}+A_{12 i} Z_{2}+B_{1 i} \tau+M_{a i}^{1} v_{a} \\
0=A_{21 i} Z_{1}+A_{22 i} Z_{2}+B_{2 i} \tau+M_{a i}^{2} v_{a} \\
y=C_{1 i} Z_{1}+C_{2 i} Z_{2}+D_{i} \tau+D_{a i} v_{a}+M_{s i} v_{s}
\end{array}\right.
$$

by finding the expression of the static variable $\mathrm{Z}_{2}$, and replacing it in (6), we obtain,

$$
\left\{\begin{array}{c}
\dot{Z}_{1}=J_{i} Z_{1}+L_{i} \tau+N_{a i} v_{a} \\
Z_{2}=S_{i} Z_{1}+V_{i} \tau+R_{a i} v_{a} \\
y=P_{i} Z_{1}+T_{i} \tau+K_{a i} v_{a}+M_{s i} v_{s}
\end{array}\right.
$$

where,

$$
\left\{\begin{array}{c}
J_{i}=A_{11 i}+A_{12 i} S_{i} \\
L_{i}=B_{1 i}+A_{12 i} V_{i} \\
N_{a i}=M_{a i}^{1}+A_{12 i} R_{a i} \\
S_{i}=-A_{22 i}^{-1} A_{21 i} \\
V_{i}=-A_{22 i}^{-1} B_{2 i} \\
R_{a i}=-A_{22 i}^{-1} M_{a i}^{2} \\
P_{i}=C_{1 i}+C_{2 i} S_{i} \\
T_{i}=D_{i}+C_{2 i} V_{i} \\
K_{a i}=D_{a i}+C_{2 i} R_{a i}
\end{array}\right.
$$

let define,

$$
v=\left(\begin{array}{l}
v_{a} \\
v_{s}
\end{array}\right)
$$

which is equivalent to the following state representation,

$$
\left\{\begin{array}{c}
\dot{Z}_{1}=J_{i} Z_{1}+L_{i} \tau+N_{i} v \\
Z_{2}=S_{i} Z_{1}+V_{i} \tau+R_{i} v \\
y=P_{i} Z_{1}+T_{i} \tau+K_{i} v
\end{array}\right.
$$


where,

$$
\left\{\begin{array}{c}
N_{i}=\left(N_{a i} 0\right) \\
R_{i}=\left(R_{a i} 0\right) \\
K_{i}=\left(K_{a i} M_{s i}\right)
\end{array}\right.
$$

then, from $(10) \rho_{\mathrm{i}}(\beta)$ can be rewritten as,

$$
\rho_{i}(\beta)=\rho_{i}\left(Z_{1}, Z_{2}=S_{i} Z_{1}+V_{i} \tau+R_{i} v\right)=\rho_{i}\left(Z_{1}, \tau, v\right)=\rho_{i}(\omega)
$$

with $\omega^{T}=\left[\begin{array}{lll}Z_{1}^{T} & \tau^{T} v^{T}\end{array}\right]$. So, the system (1) can be rewritten under the following equivalent form,

$$
\left\{\begin{array}{c}
\dot{Z}_{1}=\sum_{i=1}^{q} \rho_{i}(\omega)\left(J_{i} Z_{1}+L_{i} \tau+N_{i} v\right) \\
Z_{2}=\sum_{i=1}^{q} \rho_{i}(\omega)\left(S_{i} Z_{1}+V_{i} \tau+R_{i} v\right) \\
y=\sum_{i=1}^{q} \rho_{i}(\omega)\left(P_{i} Z_{1}+T_{i} \tau+K_{i} v\right)
\end{array}\right.
$$

Assumption 2: Assume that $v$ is considered in the following form,

$$
v=c_{0}+c_{1} t+c_{2} t^{2}+\cdots+c_{m_{f}} t^{m_{f}}
$$

where $c_{k} ; k=0,1, \ldots, m_{f}$ are real unknown constant parameters and the $\left(m_{f}+1\right)^{t h}$ time derivative of the fault is null.

Let,

$$
\varphi_{k}=v^{k-1} \text { with } k=1, \ldots, m_{f}+1
$$

then,

$$
\left\{\begin{array}{l}
\dot{\varphi}_{k}=\varphi_{k+1} \\
\dot{\varphi}_{m_{f}+1}=0
\end{array} \text { with } k=1, \ldots, m_{f}\right.
$$

thus, we rewrite the system (13) under the equivalent augmented state form as follows,

$$
\left\{\begin{array}{c}
\dot{x}_{1}=\sum_{i=1}^{q} \rho_{i}(\theta)\left(\tilde{U}_{i} x_{1}+\tilde{L}_{i} \tau\right) \\
x_{2}=\sum_{i=1}^{q} \rho_{i}(\theta)\left(\tilde{S}_{i} x_{1}+V_{i} \tau\right) \\
y=\sum_{i=1}^{q} \rho_{i}(\theta)\left(\tilde{P}_{i} x_{1}+T_{i} \tau\right)
\end{array}\right.
$$

where,

$$
\left\{\begin{array}{c}
\mathcal{X}_{1}^{T}=\left(\begin{array}{cccc}
Z_{1}^{T} & \varphi_{1}^{T} & \cdots & \varphi_{m_{f}}^{T}
\end{array}\right) \\
\chi_{2}=Z_{2} \\
\theta=\left(\begin{array}{c}
\chi_{1} \\
\tau
\end{array}\right) \\
\tilde{J}_{i}=\left(\begin{array}{ccccc}
J_{i} & N_{i} & 0 & \cdots & 0 \\
0 & 0 & I & \cdots & 0 \\
\vdots & \ddots & \ddots & \ddots & \vdots \\
0 & 0 & 0 & \cdots & I \\
0 & 0 & \cdots & 0 & 0
\end{array}\right) \\
\tilde{L}_{i}=\left(\begin{array}{lllll}
L_{i}^{T} & 0 & 0 & \cdots & 0
\end{array}\right)^{T} \\
\tilde{S}_{i}=\left(\begin{array}{lllll}
S_{i} & R_{i} & 0 & \cdots & 0
\end{array}\right) \\
\tilde{P}_{i}=\left(\begin{array}{lllll}
P_{i} & K_{i} & 0 & \cdots & 0
\end{array}\right)
\end{array}\right.
$$

\section{RESEARCH METHOD}

The following section shows the design of new structure of fuzzy observer allowing the simultaneous estimation of the unmeasurable states and unknown faults of the equivalent structure (17) of the CTSSM (1), 


$$
\left\{\begin{array}{c}
\dot{\hat{X}}_{1}=\sum_{i=1}^{q} \rho_{i}(\hat{\theta})\left(\tilde{U}_{i} \widehat{X}_{1}+\tilde{L}_{i} \tau-G_{i}(\hat{y}-y)\right) \\
\widehat{X}_{2}=\sum_{i=1}^{q} \rho_{i}(\hat{\theta})\left(\tilde{S}_{i} \widehat{X}_{1}+V_{i} \tau\right) \\
\hat{y}=\sum_{i=1}^{q} \rho_{i}(\hat{\theta})\left(\tilde{P}_{i} \widehat{x}_{1}+T_{i} \tau\right)
\end{array}\right.
$$

such that the estimated vectors of $\left(X_{1}, X_{2}\right)$ and y are denoted by $\left(\widehat{X}_{1}, \widehat{X}_{2}\right)$ and $\hat{y}$, respectively.

For $i=1, \cdots, q$ the term $G_{i}$ expresses the observer gain for the $i^{t h}$ submodel such as the estimated of the augmented vector of the states and faults tends asymptotically towards the real vector.

Defining,

$$
e=\left(\begin{array}{l}
e_{1} \\
e_{2}
\end{array}\right)=\left(\begin{array}{l}
\hat{x}_{1}-x_{1} \\
\widehat{x}_{2}-x_{2}
\end{array}\right)
$$

substituting (17) and (19) into (20) gives the following static and dynamic equations of the state estimation error,

$$
\left\{\begin{array}{c}
\dot{e}_{1}=\sum_{i=1}^{q} \rho_{i}(\hat{\theta})\left(\tilde{U}_{i} \widehat{x}_{1}+\tilde{L}_{i} \tau-G_{i}(\hat{y}-y)\right)-\sum_{i=1}^{q} \rho_{i}(\theta)\left(\tilde{U}_{i} x_{1}+\tilde{L}_{i} \tau\right) \\
e_{2}=\sum_{i=1}^{q} \rho_{i}(\hat{\theta})\left(\tilde{S}_{i} \widehat{x}_{1}+V_{i} \tau\right)-\sum_{i=1}^{q} \rho_{i}(\theta)\left(\tilde{S}_{i} x_{1}+V_{i} \tau\right)
\end{array}\right.
$$

equivalent to,

$$
\left\{\begin{array}{c}
\dot{e}_{1}=\sum_{i=1}^{q} \rho_{i}(\hat{\theta})\left(\tilde{U}_{i} e_{1}-G_{i}(\hat{y}-y)\right)-\sum_{i=1}^{q}\left(\rho_{i}(\theta)-\rho_{i}(\hat{\theta})\right)\left(\tilde{J}_{i} X_{1}+\tilde{L}_{i} \tau\right) \\
e_{2}=\sum_{i=1}^{q} \rho_{i}(\hat{\theta}) \tilde{S}_{i} e_{1}-\sum_{i=1}^{q}\left(\rho_{i}(\theta)-\rho_{i}(\hat{\theta})\right)\left(\tilde{S}_{i} x_{1}+V_{i} \tau\right)
\end{array}\right.
$$

let consider $\mathcal{F}_{i}=\tilde{J}_{i}, \tilde{L}_{i}, \tilde{S}_{i}, V_{i}$ and,

$$
\sum_{i=1}^{q}\left(\rho_{i}(\theta)-\rho_{i}(\hat{\theta})\right) \mathcal{F}_{i}=\sum_{i, j=1}^{q} \rho_{i}(\theta) \rho_{j}(\hat{\theta}) \Delta \mathcal{F}_{i j}
$$

with $\Delta \mathcal{F}_{i j}=\mathcal{F}_{i}-\mathcal{F}_{j}$

Then, by using the expression (23) the system (22) becomes,

$$
\left\{\begin{array}{c}
\dot{e}_{1}=\sum_{i=1}^{q} \rho_{i}(\hat{\theta})\left(\tilde{U}_{i} e_{1}-G_{i}(\hat{y}-y)\right)-\sum_{i, j=1}^{q} \rho_{i}(\theta) \rho_{j}(\hat{\theta})\left(\Delta \tilde{J}_{i j} x_{1}+\Delta \tilde{L}_{i j} \tau\right) \\
e_{2}=\sum_{i=1}^{q} \rho_{i}(\hat{\theta}) \tilde{S}_{i} e_{1}-\sum_{i, j=1}^{q} \rho_{i}(\theta) \rho_{j}(\hat{\theta})\left(\Delta \tilde{S}_{i j} x_{1}+\Delta V_{i j} \tau\right)
\end{array}\right.
$$

as $\sum_{i=1}^{q} \rho_{i}(\theta)=1$, we obtain,

$$
\left\{\begin{array}{c}
\dot{e}_{1}=\sum_{i, j=1}^{q} \rho_{i}(\theta) \rho_{j}(\hat{\theta})\left(\tilde{J}_{j} e_{1}-G_{j}(\hat{y}-y)\right)-\sum_{i, j=1}^{q} \rho_{i}(\theta) \rho_{j}(\hat{\theta})\left(\Delta \tilde{J}_{i j} x_{1}+\Delta \tilde{L}_{i j} \tau\right) \\
e_{2}=\sum_{i, j=1}^{q} \rho_{i}(\theta) \rho_{j}(\hat{\theta})\left(\tilde{S}_{j} e_{1}-\Delta \tilde{S}_{i j} x_{1}-\Delta V_{i j} \tau\right)
\end{array}\right.
$$

in the same way, we can get $y$ and $\hat{y}$ as follows,

$$
\left\{\begin{array}{c}
y=\sum_{i, h=1}^{q} \rho_{i}(\theta) \rho_{h}(\hat{\theta})\left(\left(\tilde{P}_{h}+\Delta \tilde{P}_{i h}\right) x_{1}+\left(T_{h}+\Delta T_{i h}\right) \tau\right) \\
\hat{y}=\sum_{i, h=1}^{q} \rho_{i}(\theta) \rho_{h}(\hat{\theta})\left(\tilde{P}_{h} \widehat{x}_{1}+T_{h} \tau\right)
\end{array}\right.
$$

with $\Delta \tilde{P}_{i h}=\tilde{P}_{i}-\tilde{P}_{h}$ and $\Delta T_{i h}=T_{i}-T_{h}$. By the substitution of (26) in (25), we get,

$$
\left\{\begin{array}{c}
\dot{e}_{1}=\sum_{i, j, h=1}^{q} \rho_{i}(\theta) \rho_{j}(\hat{\theta}) \rho_{h}(\hat{\theta})\left(\Pi_{j h} e_{1}+\Xi_{i j h} x_{1}+\nabla_{i j h} \tau\right) \\
e_{2}=\sum_{i, j=1}^{q} \rho_{i}(\theta) \rho_{j}(\hat{\theta})\left(\tilde{S}_{j} e_{1}-\Delta \tilde{S}_{i j} x_{1}-\Delta V_{i j} \tau\right)
\end{array}\right.
$$

with,

$$
\left\{\begin{array}{c}
\Pi_{j h}=\tilde{J}_{j}-G_{j} \tilde{P}_{h} \\
\Xi_{i j h}=G_{j} \Delta \tilde{P}_{i h}-\Delta \tilde{J}_{i j} \\
\nabla_{i j h}=G_{j} \Delta T_{i h}-\Delta \tilde{L}_{i j} \\
i, j, h \epsilon(1, \cdots, q)
\end{array}\right.
$$


therefore, to demonstrate the convergence of $e$ towards zero, it suffices to demonstrate that $e_{1}$ converges to zero. Considering $\tilde{e}_{1}=\left(e_{1}^{T} X_{1}^{T}\right)^{T}$, we get,

$$
\left\{\begin{array}{c}
\dot{\tilde{e}}_{1}=\sum_{i, j, h=1}^{q} \rho_{i}(\theta) \rho_{j}(\hat{\theta}) \rho_{h}(\hat{\theta})\left(\sum_{i j h} \tilde{e}_{1}+\Psi_{i j h} \tau\right) \\
e_{1}=Q \tilde{e}_{1}
\end{array}\right.
$$

with,

$$
\left\{\begin{array}{c}
\Sigma_{i j h}=\left(\begin{array}{cc}
\Pi_{j h} & \Xi_{i j h} \\
0 & \tilde{J}_{i}
\end{array}\right) \\
\Psi_{i j h}=\left(\begin{array}{c}
\nabla_{i j h} \\
\tilde{L}_{i}
\end{array}\right) \\
Q=\left(\begin{array}{ll}
I & 0
\end{array}\right)
\end{array}\right.
$$

guaranteeing the stability of (29) while attenuationg the effect of $\tau$ on $e_{1}$ is linked to the determination of the observer gains $G_{i}$ for $i=1, \cdots, q$.

Theorem: Under assumptions 1 and 2, if for the CTSSM (1) there are matrices $P_{1}, P_{2}, W_{i}$ for $i=1, \cdots, q$, and a positive scalar $\xi$ for a given $\partial>0$ which satisfy the LMIs (31), then it will be possible to determine the observer gains, that ensure the exponential convergence to zero of the estimation error,

$$
\left(\begin{array}{ccc}
\delta_{j h} & \gamma_{i j h} & \phi_{i j h} \\
\gamma_{i j h}^{T} & \kappa_{i} & P_{2} \tilde{L}_{i} \\
\phi_{i j h}^{T} & \tilde{L}_{i}^{T} P_{2} & -\xi \mathrm{I}
\end{array}\right)<0 \forall(i, j, h) \in(1, \cdots, q)^{3}
$$

with,

$$
\left\{\begin{array}{c}
\delta_{j h}=\tilde{J}_{j}^{T} P_{1}+P_{1} \tilde{J}_{j}-\tilde{P}_{h}^{T} W_{j}^{T}-W_{j} \tilde{P}_{h}+2 \partial P_{1}+I \\
\gamma_{i j h}=W_{j}\left(\tilde{P}_{i}-\tilde{P}_{h}\right)-P_{1}\left(\tilde{J}_{i}-\tilde{J}_{j}\right) \\
\phi_{i j h}=W_{j}\left(T_{i}-T_{h}\right)-P_{1}\left(\tilde{L}_{i}-\tilde{L}_{j}\right) \\
\Lambda_{i}=\tilde{J}_{i}^{T} P_{2}+P_{2} \tilde{J}_{i}+2 \partial P_{2}
\end{array}\right.
$$

the gains of the observer $G_{i}, i=1, \cdots, q$ are obtained by,

$$
G_{i}=P_{1}^{-1} W_{i}
$$

the attenuation level is,

$$
\alpha=\sqrt{\xi}
$$

Proof of Theorem: Let us consider the following quadratic Lyapunov function as follows,

$$
V\left(\tilde{e}_{1}\right)=\tilde{e}_{1}^{T} P \tilde{e}_{1}, P=P^{T}>0
$$

with,

$$
P=\left(\begin{array}{cc}
P_{1} & 0 \\
0 & P_{2}
\end{array}\right)
$$

the derivative of $V$ with respect to time is,

$$
\dot{V}=\sum_{i, j, h=1}^{q} \rho_{i}(\theta) \rho_{j}(\hat{\theta}) \rho_{h}(\hat{\theta})\left(\tilde{e}_{1}^{T}\left(\Sigma_{i j h}^{T} P+P \Sigma_{i j h}\right) \tilde{e}_{1}+\tilde{e}_{1}^{T} P \Psi_{i j h} \tau+\tau^{T} \Psi_{i j h}^{T} P \tilde{e}_{1}\right)
$$

to guarantee the stability of (29) and the boundedness of the transfer from the input $\tau$ to $e_{1}$,

$$
\frac{\left\|e_{1}\right\|_{2}}{\|\tau\|_{2}}<\alpha,\|\tau\|_{2} \neq 0
$$

we consider, 


$$
\dot{V}+e_{1}^{T} e_{1}-\alpha^{2} \tau^{T} \tau<0
$$

so, the exponential convergence of the estimation error is guaranteed if,

$$
\dot{V}+e_{1}^{T} e_{1}-\alpha^{2} \tau^{T} \tau<-2 \partial V \partial>0
$$

inserting (29) and (37) into (40) leads to the following inequality,

$$
\dot{V}=\sum_{i, j, h=1}^{q} \rho_{i}(\theta) \rho_{j}(\hat{\theta}) \rho_{h}(\hat{\theta})\left(\tilde{e}_{1}^{T} \tau^{T}\right) \Gamma_{i j h}\left(\tilde{e}_{1} \tau\right)^{T}<0
$$

with,

$$
\Gamma_{i j h}=\left(\begin{array}{cc}
\Sigma_{i j h}^{T} P+P \Sigma_{i j h}+Q^{T} Q+2 \partial P & P \Psi_{i j h} \\
\Psi_{i j h}^{T} P & -\alpha^{2} I
\end{array}\right)
$$

then, the inequality (41) is contented if,

$$
\Gamma_{i j h}<0 \forall i, j, h \in(1, \cdots, q)
$$

taking account (28), (30), (36), and the following change of variables,

$$
\left\{\begin{aligned}
W_{i} & =P_{1} G_{i} \\
\xi & =\alpha^{2}
\end{aligned}\right.
$$

we can deduce the LMIs (31) presented in the Theorem that complete the proof.

\section{RESULTS AND DISCUSSION}

To display the benefits of the suggested observer, we consider the following CTSSM which is affected by faults, at the level of actuator and sensor, and subjected to unmeasurable premise variable,

$$
\left\{\begin{array}{c}
M \dot{z}=\sum_{i=1}^{2} \rho_{i}(\beta)\left(A_{i} z+B \tau+M_{a} v_{a}\right) \\
y=C z+M_{s} v_{s}
\end{array}\right.
$$

where $z \in \mathbb{R}^{4}, \tau \in \mathbb{R}, y \in \mathbb{R}^{2}, v_{a} \in \mathbb{R}$, and $v_{s} \in \mathbb{R}$ are the vectors of states, input, output, actuator fault and sensor fault, respectively.

$$
\begin{aligned}
& A_{1}=\left(\begin{array}{cccc}
0 & 1 & 0 & 0 \\
-2.5 & -0.75 & 0 & 0.025 \\
0 & 1 & -0.4 & 0 \\
-2.5 & -0.75 & 0 & 0.075
\end{array}\right) ; A_{2}=\left(\begin{array}{cccc}
0 & 1 & 0 & 0 \\
-2.696 & -0.75 & 0 & 0.025 \\
0 & 1 & -0.4 & 0 \\
-2.696 & -0.75 & 0 & 0.075
\end{array}\right) \\
& B=\left(\begin{array}{c}
0 \\
0 \\
0 \\
-0.125
\end{array}\right) ; M_{a}=B ; C=\left(\begin{array}{cccc}
1 & 0 & 1 & 0 \\
0 & 1 & 0 & 1
\end{array}\right) ; M_{S}=\left(\begin{array}{l}
1 \\
0
\end{array}\right)
\end{aligned}
$$

$\rho_{1}(\beta)$ and $\rho_{2}(\beta)$ represent the weighting functions,

$$
\left\{\begin{array}{l}
\rho_{1}(\beta)=\frac{\beta-\beta_{\min }}{\beta_{\max }-\beta_{\min }} \\
\rho_{2}(\beta)=\frac{\beta_{\max }-\beta}{\beta_{\max }-\beta_{\min }}
\end{array}\right.
$$

where the expression of the premise variable $\beta$ is,

$$
\beta=\frac{-5-5 z_{1}^{2}}{2} \in\left[\beta_{\text {min }}, \beta_{\text {max }}\right]
$$

in order to apply the suggested fuzzy observer (19) on our application example (45), it suffices to represent it in its equivalent form (17). Thus, by using the Theorem with $\partial=0.1$, we obtain the following observer gains $G_{1}$ and $G_{2}$, 


$$
G_{1}=\left(\begin{array}{cc}
-16.2542 & 0.4992 \\
60.4899 & 19.3705 \\
443.5258 & 136.5402 \\
77.1069 & 23.0732 \\
35.2472 & -5.6930 \\
12.2730 & -3.6819
\end{array}\right) ; G_{2}=\left(\begin{array}{cc}
-16.3254 & 0.5089 \\
59.6945 & 28.7403 \\
437.8298 & 202.7100 \\
76.1530 & 34.2843 \\
35.5444 & -7.7807 \\
12.4475 & -5.1612
\end{array}\right)
$$

the simulation results are given in Figures 1 to 4 where the input signal is given by,

$$
\tau(t)=\left\{\begin{array}{c}
t-2 \text { when } t \leq 2 \\
0 \text { else }
\end{array}\right.
$$

Under Assumption 2, the trajectories of actuator and sensor fault signals, which are applied respectively during the intervals $[40,160 \mathrm{~s}]$ and $[200,320 \mathrm{~s}]$, their first order derivatives, and their estimates are shown in Figures 3 and 4.

These results demonstrate that the suggested fuzzy observer gives good performances in estimating unmeasurable states and unknown faults while catching up with unwanted variations. This approach has the benefit of being applied at the level of a large class of nonlinear systems. This is due to the fact that it is not required to know the value of the Lipschitz constant that can influence the resolution of LMIs [27], as well as without being limited by the condition of the rank between the matrices such as in [28].
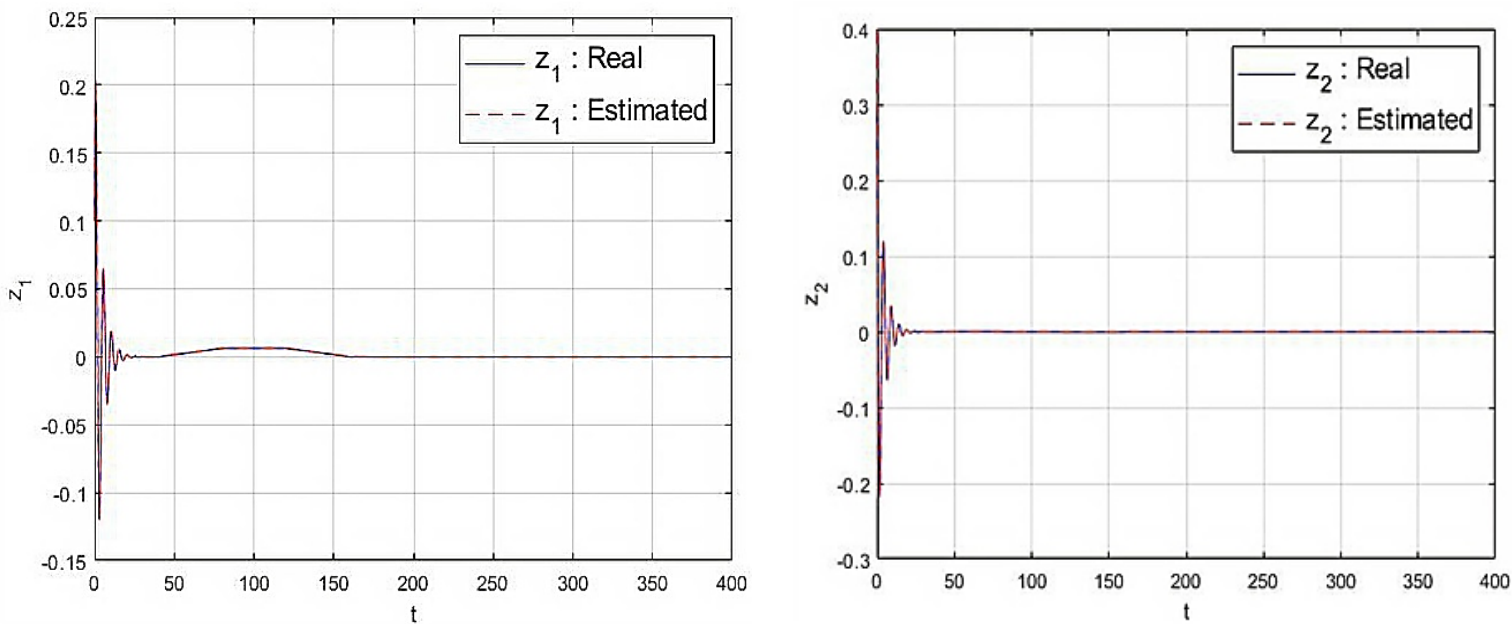

Figure $1 . \mathrm{z}_{1}$ and $\mathrm{z}_{2}$ with their estimates
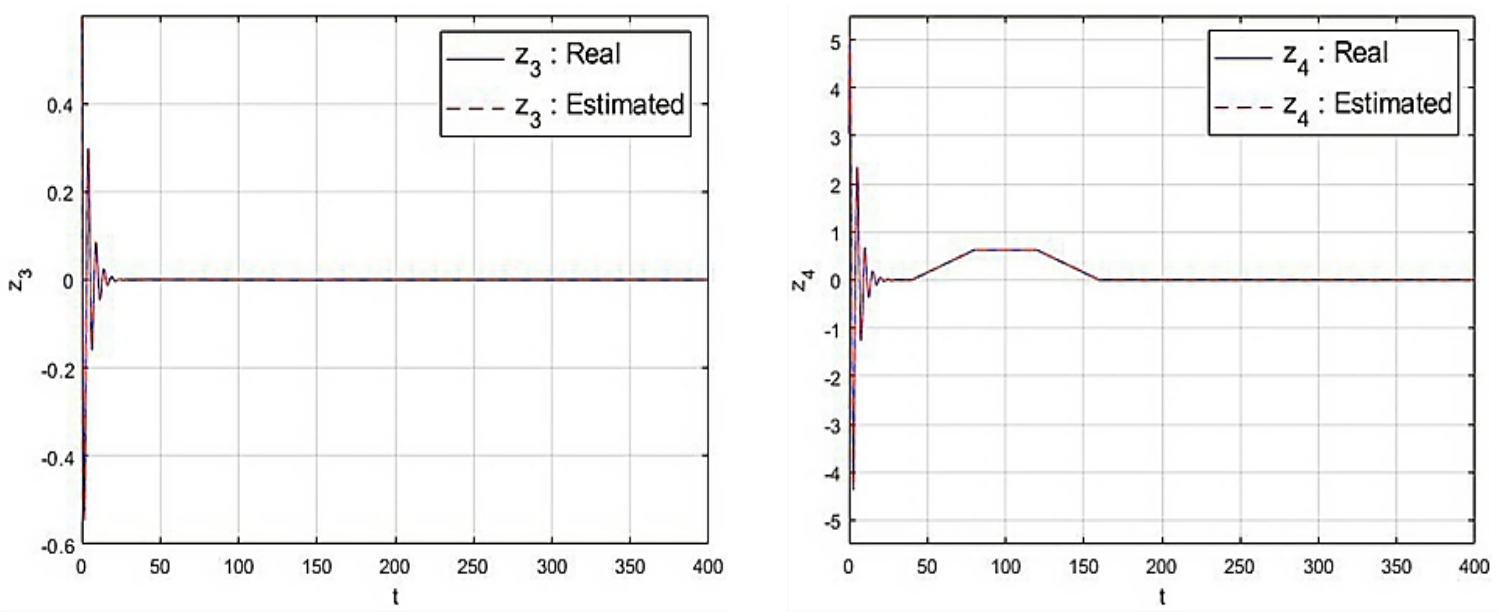

Figure $2 . z_{3}$ and $z_{4}$ with their estimates 

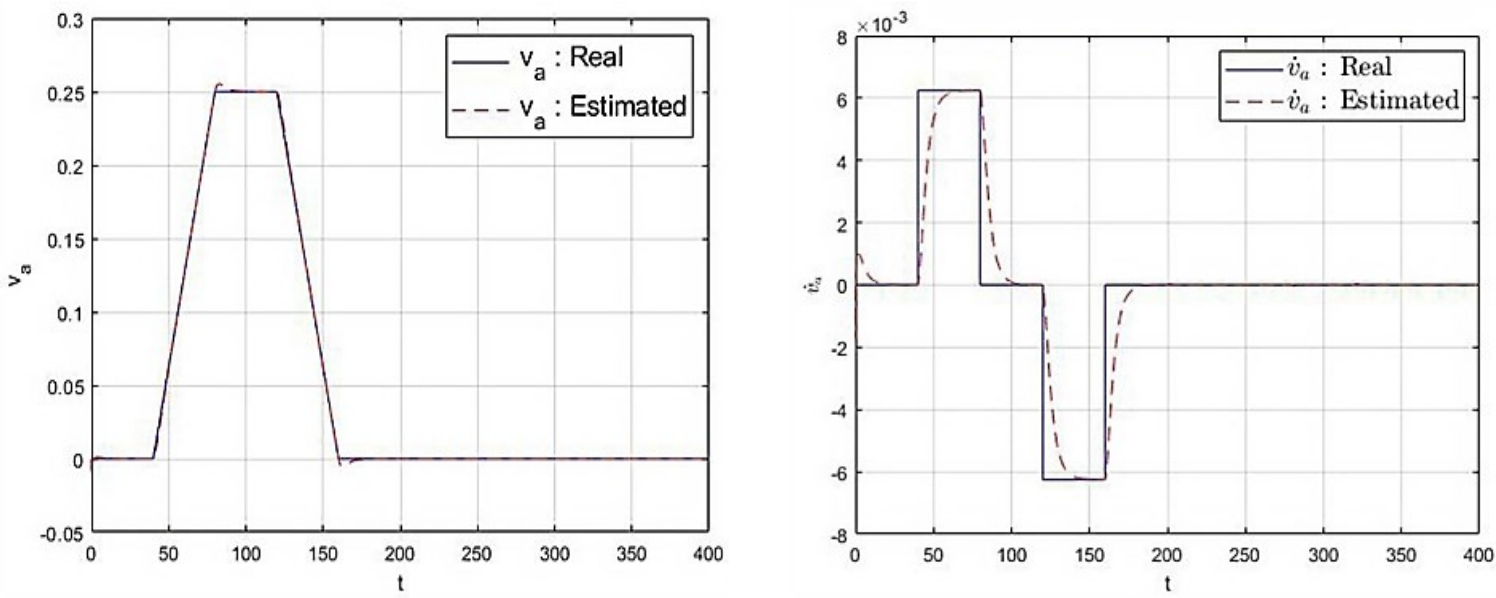

Figure 3. $v_{a}$ and $\dot{v}_{a}$ with their estimates
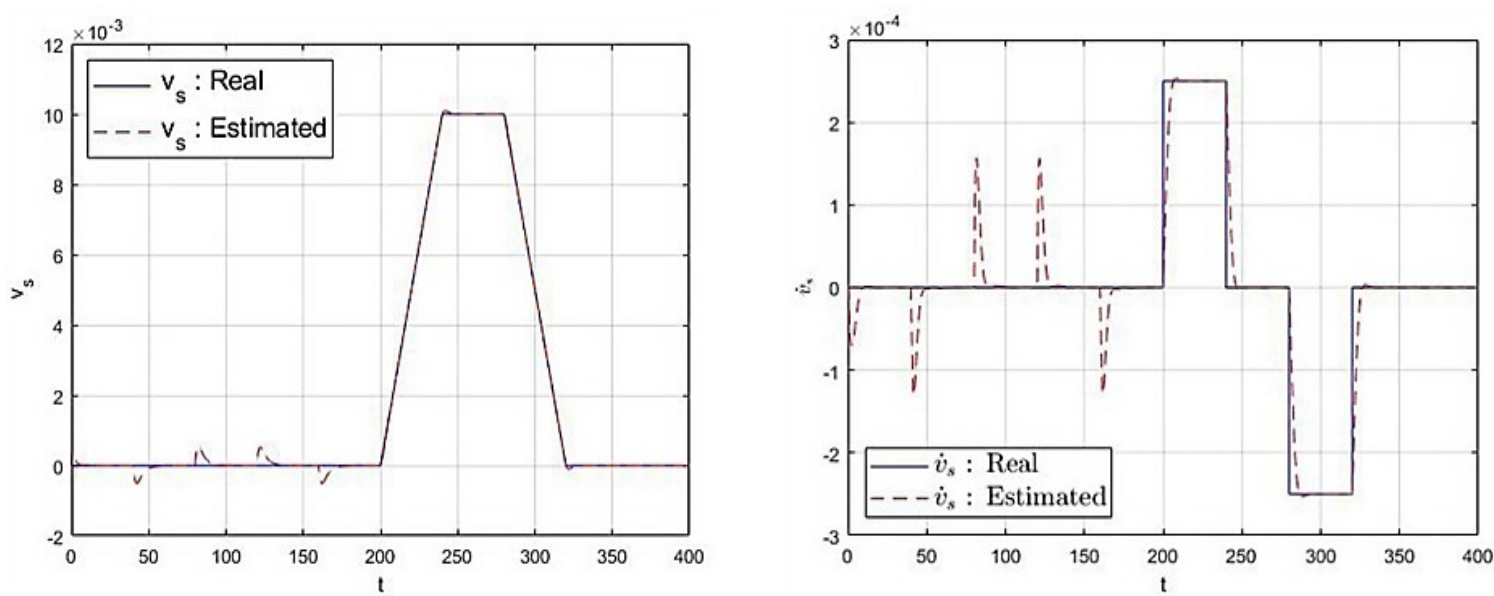

Figure 4. $v_{s}$ and $\dot{v}_{s}$ with their estimates

\section{CONCLUSION}

This work is addressed to the design of fuzzy observer for simultaneous estimation of unmeasurable states and unknown faults, for Takagi-Sugeno singular models in continuous time. The main idea of this paper is to extend the results developed in the case of measurable premise variables. The diagnostic procedure is based on the separation of static equations from dynamic ones. Using this, the determination of the static variables will be deduced from the computation of the dynamic variables. At last, an example of application is presented in order to highlight and confirm the effectiveness of the proposed approach in the estimation of the states, and the faults of actuators and sensors.

\section{REFERENCES}

[1] Y.-J. Park, S.-K S. Fan, and C.-Y. Hsu, "A Review on Fault Detection and Process Diagnostics in Industrial Processes," Processes, vol. 8, no. 9, pp. 1-26, 2020, doi: 10.3390/pr8091123.

[2] R. Arunthavanathann, F. Khan, S. Ahmed, and S. Imtiaz, "An analysis of process fault diagnosis methods from safety," Computers and Chemical Engineering, vol. 145, pp.1-20, 2021, doi:10.1016/j.compchemeng.2020.107197.

[3] L. Dai, "Singular Control Systems, " vol. 118, Springer, Germany, 1989, doi: 10.1007/BFb0002475.

[4] V. Manikandan, N. Devarajan, and K. Ramakrishnan, "A Diagnosis Approach for Parameter Deviations in Linear System Using Artificial Neural Networks," IEEE International Conference on Industrial Technology, 2006, doi: 10.1109/ICIT.2006.372282.

[5] A. N. Zhirabok, A.V. Zuev, and A.E. Shymsky, "Diagnosis of Linear Dynamic Systems: An Approach Based on Sliding Mode Observers," Automation and Remote Control, vol. 81, no. 2, pp. 211-225, 2020, doi: 10.1134/S0005117920020022.

[6] A. Valibeygi, A. Toudeshki, and K. Vijayaraghavan, "Observer-based sensor fault estimation in nonlinear systems," Proceedings of the Institution of Mechanical Engineers, Part I: Journal of Systems and Control Engineering, vol. 230, no. 8, pp. 759-777, 2016, doi:10.1109/icit.2006.372282. 
[7] E. Sobhani-Tehrani and K. Khorasani, "Fault Diagnosis of Nonlinear Systems Using a Hybrid Approach," Lecture Notes in Control and Information Sciences, vol. 383, 2009, doi:10.1007/978-0-387-92907-1.

[8] X.-P. Xie, D. Yue, and J. Park, "Robust Fault Estimation Design for Discrete-Time Nonlinear Systems via A Modified Fuzzy Fault Estimation Observer," ISA Transactions, vol. 73, pp. 22-30, 2018, doi: 10.1016/j.isatra.2017.12.007.

[9] T. Takagi and M. Sugeno, "Fuzzy identification of systems and its application to modeling and control," IEEE Trans. Syst., Man and Cybernetics, vol. 15, no. 1, pp. 116-132, 1985, doi: 10.1109/TSMC.1985.6313399.

[10] K. Tanaka and H.O. Wang, "Fuzzy control systems design and analysis: A Linear Matrix Inequality Approach," John Wiley and Sons, 2001, doi: 10.1002/0471224596.

[11] A. Mouzakitis, "Classification of Fault Diagnosis Methods for Control Systems," Measurement and Control, vol. 46, no. 10, pp. 303-308, 2013, doi: 10.1177/0020294013510471.

[12] W. Li, H. Li, S. Gu, and T. Chen, "Process fault diagnosis with model- and knowledge-based approaches: Advances and opportunities," Control Engineering Practice, vol. 105, pp. 1-17, 2020, doi: 10.1016/j.conengprac.2020.104637.

[13] Z. Gao, C. Cecati, and S. X. Ding, "A survey of fault diagnosis and fault-tolerant techniques-part I: Fault diagnosis with modelbased and signal-based approaches," IEEE Transactions on Industrial Electronics, vol. 62, no. 6, pp. 3757-3767, 2015, doi: 10.1109/TIE.2015.2417501.

[14] Z. Gao, C. Cecati, and S. X. Ding, "A survey of fault diagnosis and fault-tolerant techniques-part II: Fault diagnosis with knowledge-based and hybrid/active approaches," IEEE Transactions on Industrial Electronics, vol. 62, no. 6, pp. 3768-3774, 2015, doi: 10.1109/TIE.2015.2419013.

[15] K. O. Omali, M. N. Kabbaj, and M. Benbrahim, "Actuator fault detection and isolation for robot manipulator using higher order sliding mode observers," Indonesian Journal of Electrical Engineering and Computer Science (IJEECS), vol. 17, no. 3, pp. 11501156, 2020, doi: 10.11591/ijeecs.v17i3.pp1150-1156.

[16] T. Sellami, H. Berriri, S. Jelassi, A. M. Darcherif, and M. F. Mimouni, "Sliding mode observers-based fault detection and isolation for wind turbine-driven induction generator," International Journal of Power Electronics and Drive Systems (IJPEDS), vol. 8, no. 3, pp. 1345-1358, 2017, doi: 10.11591/ijpeds.v8i3.pp1345-1358.

[17] N. R. B. Hatem, M. Mostefai, and O. E. K. Aktouf, "Extended kalman observer based sensor fault detection," International Journal of Electrical and Computer Engineering (IJECE), vol. 9, no. 3, pp. 1546-1552, 2019, doi: 10.11591/ijece.v9i3.pp15461552 .

[18] D. Saoudi, C. Mechmeche, M. Chadli, and N. B. Braiek, "Robust residual generator design for Takagi-Sugeno fuzzy bilinear system subject to unknown inputs," IFAC Proceedings, vol. 45, pp. 1023-1028, 2012, doi: 10.3182/20120829-3-MX-2028.00191.

[19] Y. Wu and J. Dong, "Fault detection for T-S fuzzy systems with partly unmeasurable premise variables," Fuzzy Sets and Systems, vol. 338, pp. 136-156, 2017, doi: 10.1016/j.fss.2017.06.006.

[20] C. Martinez Garcia,V. Puig, C. M. Astorga-Zaragoza, and G. L. Osorio-Gordillo, "Robust Fault Estimation based on Interval Takagi-Sugeno Unknown Input Observer," International Federation of Automatic Control, vol. 51, no. 24, pp. 508-514, 2018, doi: 10.1016/j.ifacol.2018.09.624.

[21] W. Zhang, Z. Wang, T. Raïssi, and Y. Shen, "A state augmentation approach to interval fault estimation for descriptor systems," European Journal of Control, vol. 51, pp. 19-29, 2019, doi: 10.1016/j.ejcon.2019.06.006.

[22] M. A. Eissa, A. Sali, M. K. Hassan, A. M. Bassiuny, and R. R. Darwish, "Observer-Based Fault Detection with Fuzzy Variable Gains and Its Application to Industrial Servo System," IEEE Access, vol. 8, pp. 131224-131238, 2020, doi: 10.1109/ACCESS.2020.3010125.

[23] D. Yang, Y. Wang, and Z. Chen, "Robust fault diagnosis and fault tolerant control for PEMFC system based on an augmented LPV observer," International Journal of Hydrogen Energy, vol. 45, no. 24, pp. 13508-13522, 2020, doi: 10.1016/j.ijhydene.2020.03.063.

[24] E. Bernardi and E. J. Adam, "Observer-based fault detection and diagnosis strategy for industrial processes," Journal of the Franklin Institute, vol. 357, no. 14, pp. 10054-10081, 2020, doi: 10.1016/j.jfranklin.2020.07.046.

[25] K. Ouarid, A. E. Assoudi, J. Soulami, and E. H. E. Yaagoubi, "Observer Design for Simultaneous State and Fault Estimation for a Class of Continuous-time Implicit Linear Models," IEEE The International Conference of Computer Science and Renewable Energies, vol. 229, pp. 1-6, 2019, doi: 10.1109/ICCSRE.2019.8807633.

[26] K. Ouarid, A. E. Assoudi, J. Soulami, and E. H. E. Yaagoubi, "Design of Fuzzy Observer for a Class of Takagi-Sugeno Descriptor Models to Simultaneously Estimate States and Faults," Journal of Advanced Research in Dynamical and Control Systems, vol. 12, no. 5, pp. 239-250, 2020, doi: 10.5373/JARDCS/V12SP5/20201754.

[27] M. Ouzaz, A. E. Assoudi, J. Soulami, and E. H. E. Yaagoubi, "Simultaneous state and fault estimation for Takagi-Sugeno implicit models with Lipschitz constraints," International Journal of Optimization and Control: Theories and Applications, vol. 11, no. 1, pp. 100-108, 2021, doi: 10.11121/ijocta.01.2021.00877.

[28] K. A. Daraou, J. Soulami, A. E. Assoudi, and E. H. E. Yaagoubi, "State and Fault Observer Design for a Class of Takagi-Sugeno Descriptor Models," in 1st International Conference on Innovative Research in Applied Science, Engineering and Technology, Marrakech, Morocco, pp. 1-6, 2020, doi: 10.1109/IRASET48871.2020.9091990.

\section{BIOGRAPHIES OF AUTHORS}

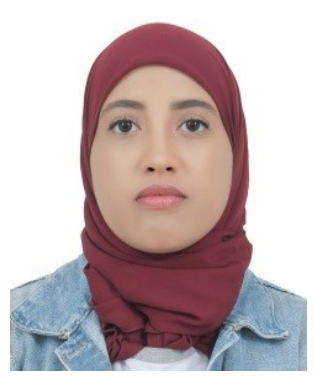

Kaoutar Ouarid (D) Bd SC $\mathrm{P}$ received the M.Sc. degree in Electrical Engineering from Faculty of Science and Technology Marrakech, Morocco, in 2016. Currently, she is working toward the PhD degree at Faculty of Science Ain Chock (FSAC), in Hassan II University of Casablanca, Morocco. Her research interests include observer design, nonlinear systems, Takagi-Sugeno fuzzy systems, fuzzy control, and fault diagnosis. She can be contacted at email: kaoutar.ouarid@gmail.com. 

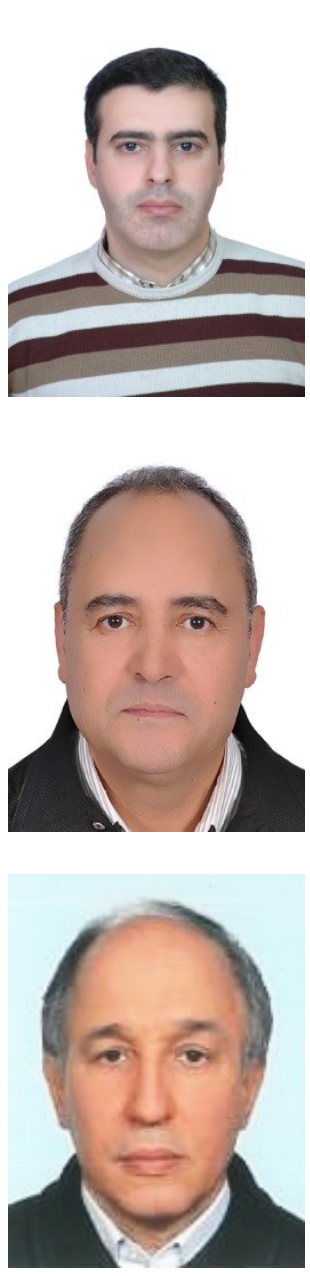

Mohamed Essabre (iD) $\mathrm{SC}$ P received the $\mathrm{PhD}$ in Engineering Science-Electrical Engineering in 2015 from the National Higher School of Electricity and Mechanics (ENSEM), Hassan II University of Casablanca, Morocco. His research interests include nonlinear control and state observer theory. Currently, he is an Associate Professor at the Department of Electrical Engineering, Faculty of Science and Technology Mohammedia, Hassan II University of Casablanca, Morocco. He can be contacted at email: mohamed.essabre@fstm.ac.ma.

Abdellatif El Assoudi (iD 81 SC P is a professor at the department of Electrical Engineering in National Higher School of Electricity and Mechanics (ENSEM), in Hassan II University of Casablanca (Morocco). His research interests focus in nonlinear implicit model, nonlinear observer design, unknown input observer design, fault detection, Takagi-Sugeno fuzzy control. He can be contacted at email: a.elassoudi@ensem.ac.ma.

El Hassane El Yaagoubi (D) SC $\mathrm{SP}$ is a professor at the department of Electrical Engineering in National Higher School of Electricity and Mechanics (ENSEM), in Hassan II University of Casablanca (Morocco). His areas of interest include Nonlinear implicit model, Nonlinear observer design, Unknown Input Observer Design, fault detection, Takagi-Sugeno fuzzy control. He can be contacted at email: h.elyaagoubi@ensem.ac.ma. 